\title{
Optimization of Trajectory Correction Scheme for Guided Mortar Projectiles
}

\author{
Yongwei Zhang, Min Gao, Suochang Yang, and Dan Fang \\ Electronic Engineering Department, Shijiazhuang Mechanical Engineering College, No. 97 Heping West Road, Shijiazhuang, \\ Hebei 050003, China \\ Correspondence should be addressed to Yongwei Zhang; yongweil112@sina.com
}

Received 16 September 2015; Revised 29 October 2015; Accepted 1 November 2015

Academic Editor: Mahmut Reyhanoglu

Copyright (c) 2015 Yongwei Zhang et al. This is an open access article distributed under the Creative Commons Attribution License, which permits unrestricted use, distribution, and reproduction in any medium, provided the original work is properly cited.

Guidance with traditional trajectory correction scheme usually starts from the trajectory apex time to reduce drag penalties early in flight; however, this method cannot get the max trajectory correction capability of canards according to our analysis. This paper presents an optimized trajectory correction scheme by taking different control phases of canards in ballistic ascending segment and ballistic descending segment. Simulation indicates that the optimized trajectory correction can improve the trajectory correction capability greatly. The result of an example trajectory and Monte Carlo simulations with the predictive guidance law and the trajectory tracking guidance law testifies the effectiveness of the optimized trajectory correction scheme.

\section{Introduction}

Firing accuracy of the guided projectiles can be dramatically improved by outfitting with a suitable trajectory correction system. The commonly used tools are impulse thrusters [1-3], inertial loads $[4,5]$, drag brakes $[6,7]$, fixed canards [8-10], and moveable canards [11-15].

Research and development on the use of canards have been going on for decades. Rogers and Costello [11] have presented a design of a canard-controlled mortar projectile using a bank-to-turn concept; the smart mortar is equipped with a set of two reciprocating fixed-angle roll canards and a set of two reciprocating fixed-angle maneuver canards. An active control system is designed to perform trajectory corrections and Monte Carlo simulations demonstrate the control system effectiveness in reducing dispersion error. Cooper et al. [13] have extended standard projectile linear theory to account for aerodynamic asymmetries caused by actuating canards, and the extended linear-projectile theory offers a tool to address flight stability of projectiles with aerodynamic configuration asymmetries. Spagni et al. [14] have characterized the system equilibrium point manifold in terms of a minimal vector of scheduling variables for a class of reciprocating canard-guided artillery munitions, giving rise to a discussion concerning the canard size and position for maneuverability optimization. Theodoulis et al. [15] have presented a complete design concerning the guidance and autopilot modules for a class of spin-stabilized fin-controlled projectiles.

The guidance usually starts from the trajectory correction apex time to reduce drag penalties early in flight in the studies of guided projectiles with canards [11-15]; however, this trajectory correction scheme cannot get the max trajectory correction capability of canards according to our analysis.

In this paper, an optimized trajectory correction scheme is put forward to maximize the trajectory correction capability and improve firing accuracy of guided mortar projectiles. Section 2 presents the trajectory model of the guided mortar projectile. Section 3 presents the analysis and optimization of trajectory correction capability. Section 4 puts forward the optimized trajectory correction scheme. Section 5 describes the result of simulations, and conclusion is provided in Section 6.

\section{6-DOF Trajectory Model}

The mortar projectile configuration used in this study is a representative $120 \mathrm{~mm}$ mortar projectile, $0.9 \mathrm{~m}$ long, finstabilized. The initial velocity is $280 \mathrm{~m} / \mathrm{s}$; the projectile weight, mass center location from the nose tip, roll inertia, and pitch 


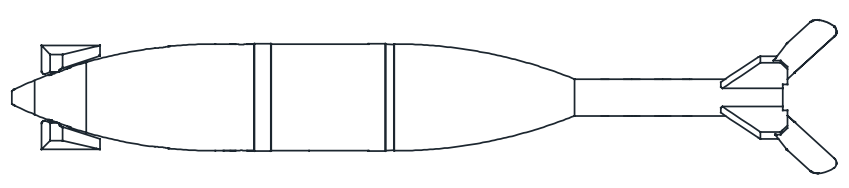

FIGURE 1: Schematic of the guided mortar projectile.

inertia are $15.0 \mathrm{~kg}, 0.387 \mathrm{~m}, 0.0261 \mathrm{~kg}-\mathrm{m}^{2}$, and $0.70 \mathrm{~kg}-\mathrm{m}^{2}$, respectively. Figure 1 shows schematic of the guided mortar projectile; the canards are mounted on the nose of the guided mortar projectile.

Figure 2 shows schematic of canards, which are driven by one actuator. Figures 2(a) and 2(b) show schematic of canards with no deflection, and Figures 2(c) and 2(d) show schematic of canards with a negative deflection angle, as shown in Figure 2; the negative deflection angle produces positive roll acceleration. The range of canards deflection angle is set as $-15 \sim 15 \mathrm{deg}$. to ensure the fight stability of the guided mortar projectile.

Figure 3 shows schematic of forces on the guided mortar projectile and the forces applied on the guided mortar projectile including weight force and aerodynamic force; the deflection angle of canards is adjusted to change the aerodynamic force for trajectory correction in flight.

In Figure 3, $\alpha$ is the attack angle, $G$ is the weight force, and $F$ is the aerodynamic force.

The numerical simulation is based on a rigid body sixdegree-of-freedom model typically utilized in flight dynamic analysis of mortar projectiles $[3,16]$. The translational kinetic differential equations are given by

$$
\begin{array}{r}
\frac{d V}{d t}=G_{x_{2}}+F_{x_{2}}, \\
V \frac{d \theta}{d t}=G_{y_{2}}+F_{y_{2}}, \\
-V \cos \theta \frac{d \psi_{v}}{d t}=G_{z_{2}}+F_{z_{2}} .
\end{array}
$$

The applied forces in (1) consist of weight force $(G)$ and aerodynamic force $(F)$, expressed in aeroballistic reference frame. $V, \psi_{v}$, and $\theta$ are the velocity, trajectory azimuth angle, and trajectory incline angle, respectively.

The rotational kinetic differential equations are given by

$$
\begin{aligned}
{\left[\begin{array}{l}
J_{x_{4}} \frac{d \omega_{x_{4}}}{d t} \\
J_{y_{4}} \frac{d \omega_{y_{4}}}{d t} \\
J_{z_{4}} \frac{d \omega_{z_{4}}}{d t}
\end{array}\right]=} & {\left[\begin{array}{c}
M_{x_{4}}+M_{x_{4}}^{\prime} \\
M_{y_{4}}+M_{y_{4}}^{\prime} \\
M_{z_{4}}+M_{z_{4}}^{\prime}
\end{array}\right]-\left[\begin{array}{c}
0 \\
\left(J_{x_{4}}-J_{z_{4}}\right) \omega_{x_{4}} \omega_{z_{4}} \\
\left(J_{y_{4}}-J_{x_{4}}\right) \omega_{x_{4}} \omega_{y_{4}}
\end{array}\right] } \\
& +\left[\begin{array}{c}
0 \\
-J_{z_{4}} \omega_{z_{4}} \frac{d \gamma}{d t} \\
J_{y_{4}} \omega_{y_{4}} \frac{d \gamma}{d t}
\end{array}\right] .
\end{aligned}
$$

The applied moments in (2) contain contributions from steady air loads, denoted by $M$, and unsteady air loads, denoted by $M^{\prime}$, expressed in quasibody reference frame. $J_{x_{4}}$, $J_{y_{4}}$, and $J_{z_{4}}$ are components of the transverse moment of inertia. $\omega_{x_{4}}, \omega_{y_{4}}$, and $\omega_{z_{4}}$ are components of the angular rate vector; $\gamma$ is the Euler roll angle.

The translational kinematic equations are given by

$$
\begin{aligned}
& \frac{d x}{d t}=V \cos \theta \cos \psi_{v}, \\
& \frac{d y}{d t}=V \sin \theta \\
& \frac{d z}{d t}=-V \cos \theta \sin \psi_{v} .
\end{aligned}
$$

In (3), $x, y$, and $z$ are position vector components of the center of mass, expressed in the inertial reference frame.

The rotational kinematic equations are given by

$$
\begin{aligned}
& \frac{d \vartheta}{d t}=\omega_{z_{4}}, \\
& \frac{d \psi}{d t}=\frac{1}{\cos \vartheta} \omega_{y_{4}}, \\
& \frac{d \gamma}{d t}=\omega_{x_{4}}-\omega_{y_{4}} \tan \vartheta .
\end{aligned}
$$

In (4), $\vartheta$ is the pitch angle and $\psi$ is the yaw angle.

The angles in $(1) \sim(4)$ have the relation expressed as follows:

$$
\begin{aligned}
& \beta=\arcsin \left[\cos \theta \sin \left(\psi-\psi_{v}\right)\right], \\
& \alpha=\vartheta-\arcsin \left(\frac{\sin \theta}{\cos \beta}\right), \\
& \gamma_{v}=\arcsin (\tan \theta \tan \beta) .
\end{aligned}
$$

In (5), $\alpha$ is the attack angle and $\beta$ is the sideslip angle.

Equations (1) (5) constitute the rigid body six-degree-offreedom model for guided mortar projectiles, which can be solved by the fourth-order Runge-Kutta algorithm.

\section{Analysis and Optimization of Trajectory Correction Capability}

3.1. Analysis of Trajectory Correction Capability. The guided mortar projectile is fin-stabilized, which will roll slowly in the flight progress, and Figure 4 plots the roll rate of the guided mortar projectile. The canards used in the guided mortar projectiles are a pair of proportional electrokinetic canards, the control method of the canards lets the deflection angle of canards follow a sinusoidal signal of the projectile roll angle, and the strength and direction of aerodynamic force accused by canards are changed through the change of the amplitude and phase of the sinusoidal signal. The control progress of canards deflection angle is shown in Figure 5.

As shown in Figure $5, \delta_{t}$ is the canards deflection angle, $\delta_{0}$ is the amplitude of canards deflection angle, $\phi$ is the phase 


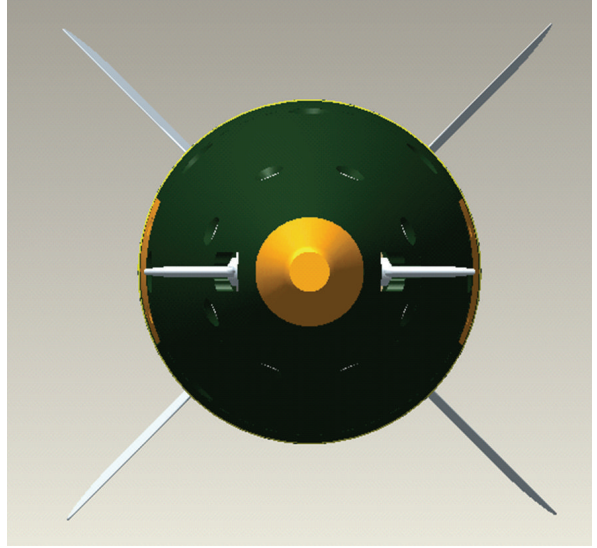

(a) Canards with no deflection angle viewed from projectile top

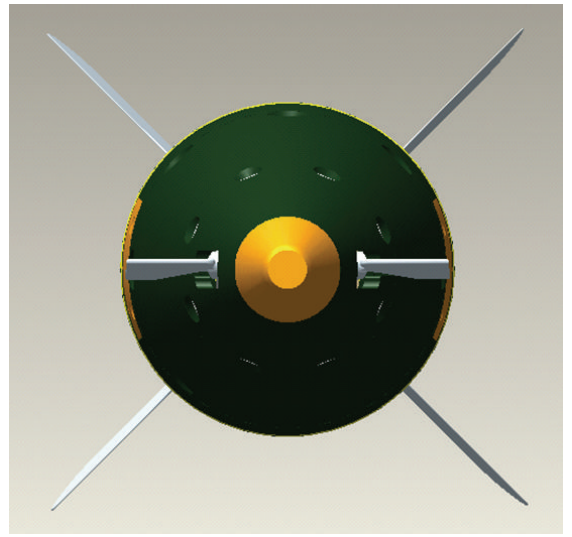

(c) Canards with negative deflection angle viewed from projectile top

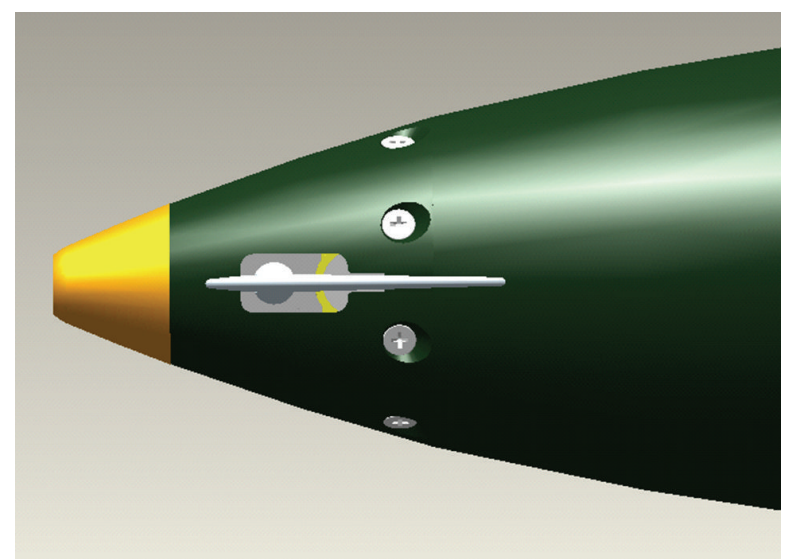

(b) Canards with no deflection angle viewed from projectile left

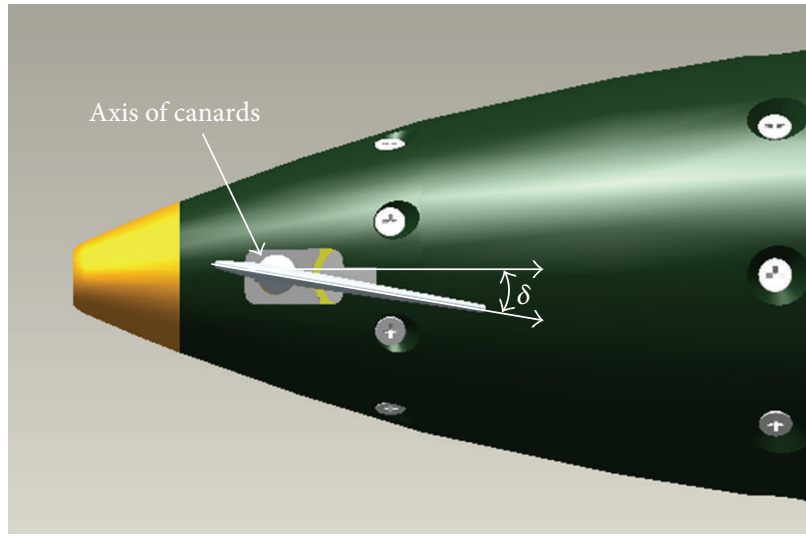

(d) Canards with negative deflection angle viewed from projectile left

Figure 2: Schematic of canards.

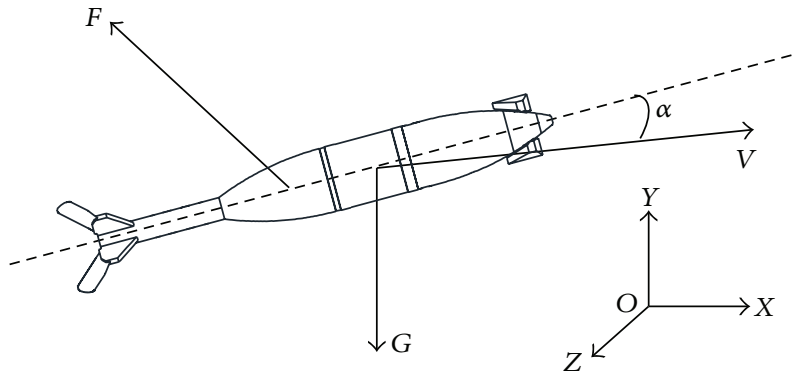

FIGURE 3: Schematic of forces on guided mortar projectiles.

of the sinusoidal signal, which is named as canards control phase, and $\gamma$ is the roll angle. The variables in Figure 5 have the following relation:

$$
\delta_{t}=\delta_{0} \cdot \sin \left(\gamma+\frac{\pi}{2}-\phi\right) .
$$

As shown in (6), trajectory correction capability will be influenced by the factors like canards control amplitude $\delta_{0}$, canards control phase $\phi$, and control start time $t_{0}$. To investigate the correction performance of canards, some simulations of the guided mortar have been done by numerical integration of the equations described above using a fourth-order Runge-Kutta algorithm. The mortar projectile is launched at sea level toward a target on the ground with altitude and cross range equal to zero at a range of $2000 \mathrm{~m}$. The traditional method to characterize trajectory correction capability is exerting control normal force in a given roll orientation from a given time [17], so the method to calculate trajectory correction capability in this study is as follows: firstly, set the launching elevation angle, simulate the uncontrolled trajectory, and make sure that the range of uncontrolled trajectory equals $2000 \mathrm{~m}$. Secondly, set the same launching elevation angle and simulate the controlled trajectory with different parameters such as different control start times, different canards control phases, and different canards control amplitudes. Thirdly, calculate the trajectory correction capability through comparing the impact point of the controlled trajectory to the impact point of the uncontrolled trajectory. For example, the launching elevation angle is set as $78.86 \mathrm{deg}$. to simulate the uncontrolled trajectory; on the other hand, canards control amplitude is set as $15 \mathrm{deg}$., the canards control phase is set as $180 \mathrm{deg}$., and control start time is set as $20 \mathrm{~s}$ for the controlled trajectory. The contrast of 


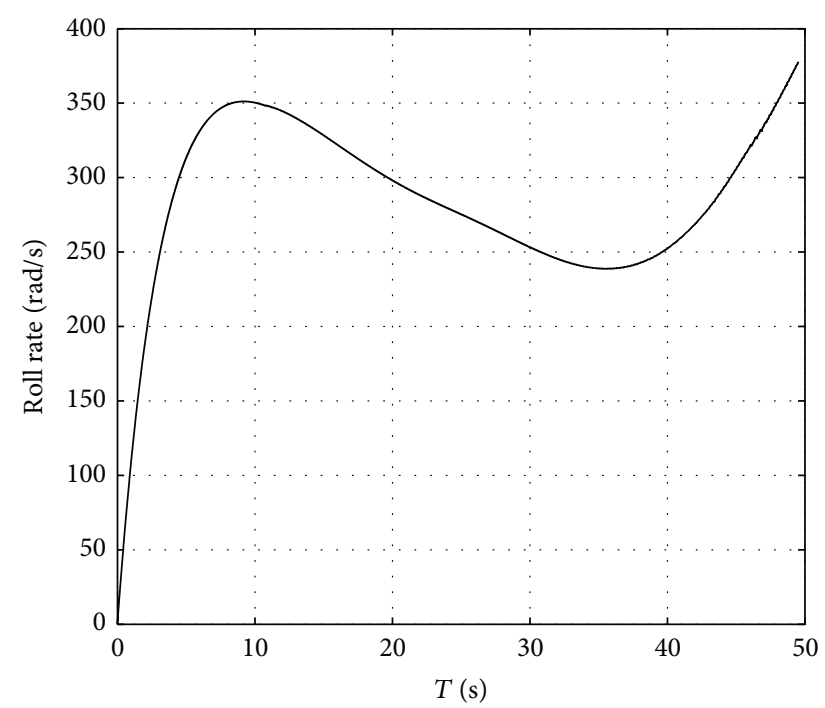

Figure 4: Roll rate versus time.

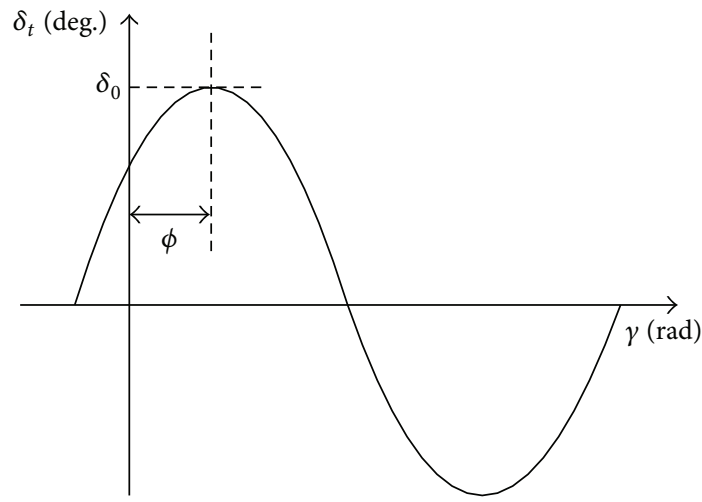

Figure 5: Schematic of canards deflection angle.

the controlled trajectory and uncontrolled trajectory is shown in Figure 6; the range correction capability is about $154 \mathrm{~m}$ according to the method of calculating trajectory correction capability described above.

The simulations with different control parameters were done with the method described above; in these simulations, $\delta_{0}$ is set as max value $(15 \mathrm{deg}$.) to get the max trajectory correction capability and $\phi$ is set as $180 \mathrm{deg}$. or $270 \mathrm{deg}$. to get the change laws of range and cross range correction capability. The launching elevation angle is 78.86 deg.; the simulation result of range and cross range correction capability is shown in Figure 7.

As shown in Figure 7, range correction capability and cross range correction capability have different change laws. The range correction capability increases as the control start time increases when the control start time is earlier than the trajectory apex time $(23.3 \mathrm{~s})$, while the range correction capability decreases as the control start time increases when the control start time is later than the trajectory apex time. At the same time, the cross range correction capability decreases

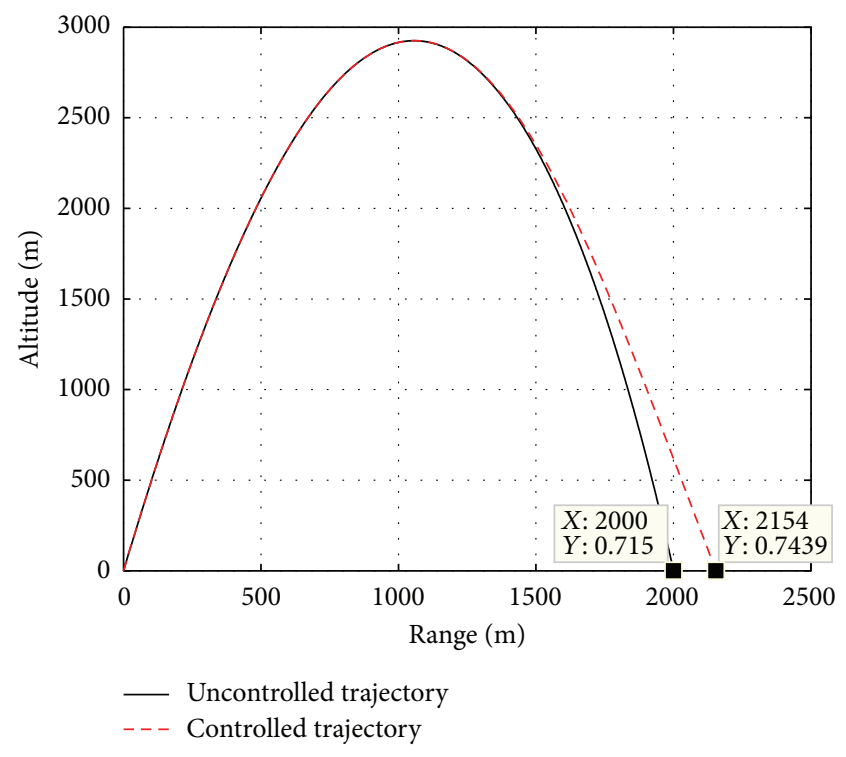

FIGURE 6: Altitude versus range.

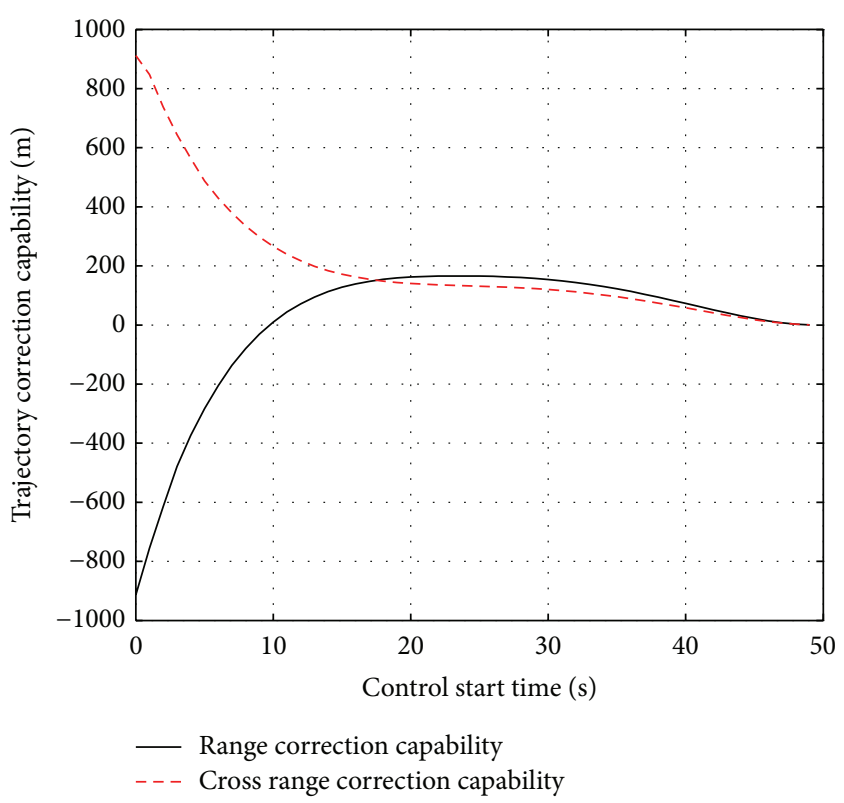

FIgURE 7: Trajectory correction capability versus control start time.

as the control start time increases whether the control start time is earlier than the trajectory apex time or not.

The behavior of range correction capability and cross range correction capability has a physical reason. Supposing that the canards control phase is set as 180 deg., the projectile will subject aerodynamic force causing canards deflection, denoted by $F$, the direction of $F$ is nearly perpendicular to the velocity direction of the projectile, $F$ can be decomposed into $F_{x}$ and $F_{y}$, as shown in Figure 8, in ballistic ascending segment, $F_{x}$ is negative, which will shorten the projectile range, while $F_{y}$ is positive, which will extend the projectile range, the impact of $F_{x}$ and $F_{y}$ is contradictory, the impact of $F_{x}$ is bigger than the impact of $F_{y}$, and a range correction 


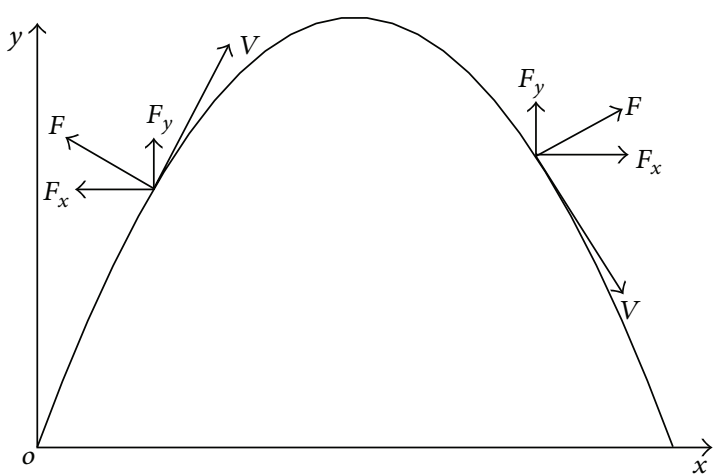

FIGURE 8: Schematic of aerodynamic force caused by canards deflection.

capability loss will be caused; the earlier the control start time, the bigger the range correction capability loss; therefore the range correction capability increases as the control start time increases in the ballistic ascending segment. In ballistic descending segment, $F_{x}$ is positive, which will extend the projectile range, and $F_{y}$ is positive, which will also extend the projectile range; then the range correction distance is mainly influenced by the time to go; therefore, the range correction decreases gradually with the increase of control start time in ballistic descending segment. In the horizontal plane, $F_{z}$ is the only component decomposed from $F$, so the cross range correction capability is mainly influenced by the time to go; hence the cross range correction capability decreases gradually with the decrease of time to go. Therefore, the range correction capability and cross range correction capability have the change trends shown in Figure 7. The max range correction capability is $166 \mathrm{~m}$, and the max cross range correction capability is $912 \mathrm{~m}$.

3.2. Optimization of Trajectory Correction Capability. As discussed above, range correction capability increases as the control start time increases in ballistic ascending segment because a range correction capability loss will be caused when the control start time is earlier than the trajectory apex time. An optimized canards control method is put forward to increase the range correction capability; this method is setting different canards control phases in ballistic ascending segment and ballistic descending segment for range correction. Take extending projectile range as an example, the canards control phase is set as 0 deg. in ballistic ascending segment, and the canards control phase is set as $180 \mathrm{deg}$. in ballistic descending segment, the aerodynamic force on the projectile caused by canards deflection is shown in Figure 9, in ballistic ascending segment, $F$ is decomposed into $F_{x}$ and $F_{y}, F_{x}$ is positive, which will extend the projectile range, while $F_{y}$ is negative, which will shorten the projectile range, and the impact of $F_{x}$ and $F_{y}$ is contradictory: the impact of $F_{x}$ is bigger than the impact of $F_{y}$; then the range correction capability loss can be avoided.

The optimized method to increase the range correction capability is expressed in the following equations.

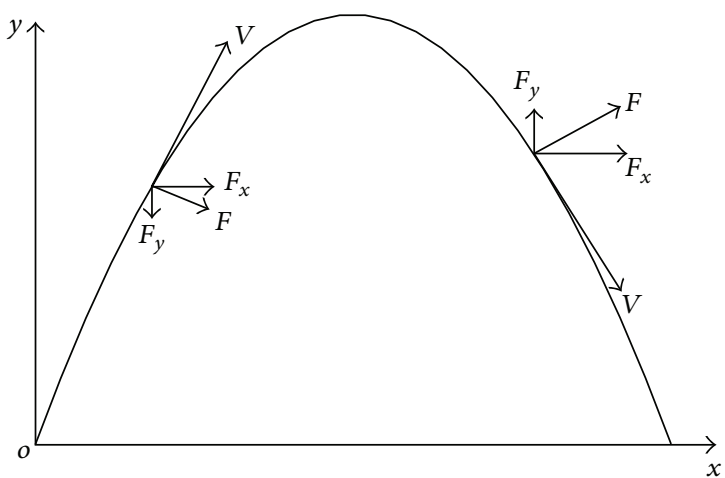

FIGURE 9: Schematic of aerodynamic force caused by canards deflection (optimized method).

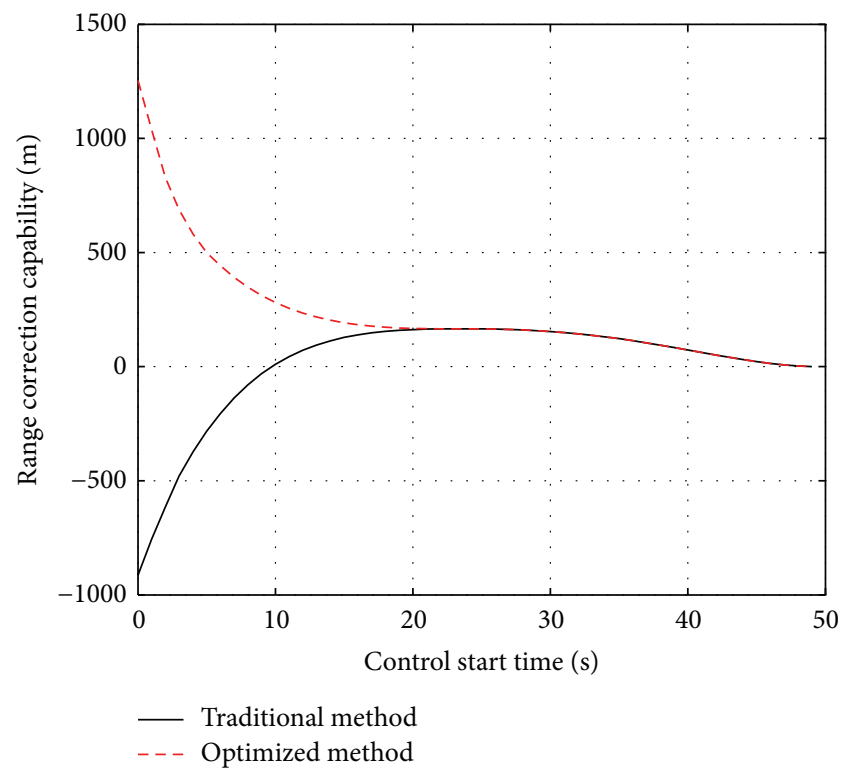

FIgURE 10: Contrast of range correction capability with traditional method and optimized method.

The method to extend projectile range is

$$
\begin{array}{ll}
\phi=0 & t<t_{\text {apex }}, \\
\phi=\pi & t \geq t_{\text {apex }} .
\end{array}
$$

The method to shorten projectile range is

$$
\begin{aligned}
& \phi=\pi \quad t<t_{\text {apex }}, \\
& \phi=0 \quad t \geq t_{\text {apex }} .
\end{aligned}
$$

Taking extending range as an example, $\delta_{0}$ is set as the max value ( $15 \mathrm{deg}$.), and the control start time is set as $0 \mathrm{~s}$; the launching elevation angle is $78.86 \mathrm{deg}$. The simulation result of range correction capability with traditional method and optimized method is shown in Figure 10. The range correction capability with optimized method decreases as the control start time increases; the max range correction capability is improved from $166 \mathrm{~m}$ to $1253 \mathrm{~m}$ with the optimized 


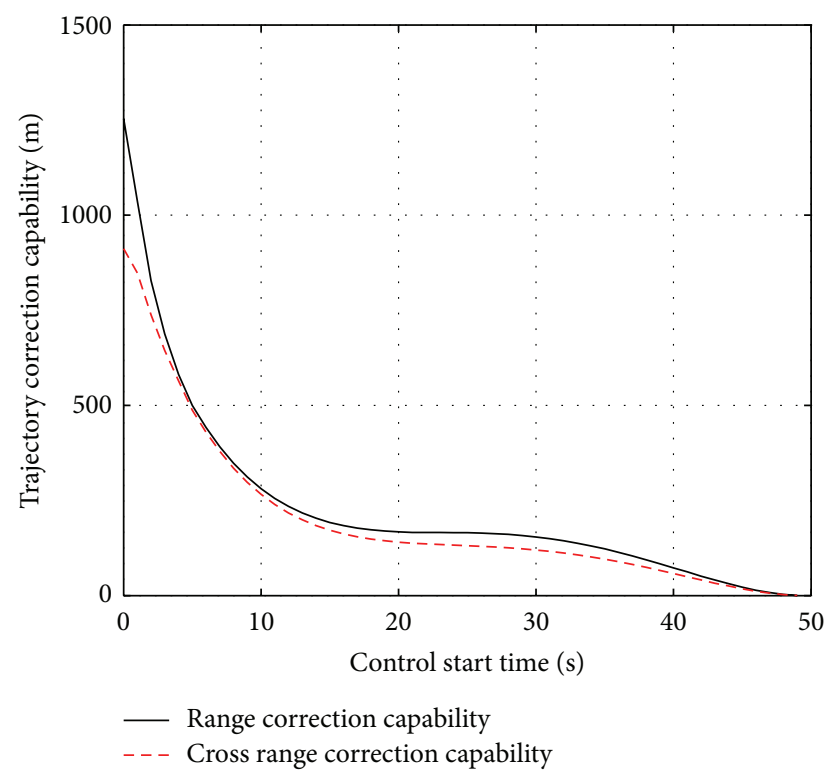

FIGURE 11: Trajectory correction capability versus control start time (using the optimized method).

method. Then trajectory correction capability with optimized method is shown in Figure 11, the range correction capability and cross range correction capability have the same change laws, range and cross range correction capability decreases as the control start time increases, the max range correction capability is $912 \mathrm{~m}$, and the max range correction capability is $1253 \mathrm{~m}$.

\section{Optimization of Trajectory Correction Scheme}

A predictive guidance law and a trajectory tracking guidance law are introduced for the guidance of the guided mortar projectiles, and the optimization of trajectory correction scheme is done for the two guidance laws $[18,19]$.

4.1. Optimization of Trajectory Correction Scheme for the Predictive Guidance Law. The schematic of predictive guidance is shown in Figure 12; the position of the target in inertial reference frame is $T$, while the position of the predictive impact point in inertial reference frame is $I$; then the predictive impact point deviation between the predictive impact point and the target is $(\Delta L, \Delta H)$, where $\Delta L$ is longitudinal impact point deviation and $\Delta H$ is horizontal impact point deviation. The objective of predictive guidance scheme is making $\Delta L$ and $\Delta H$ equal to zero through the control of the canards.

In Figure 12, $\varepsilon$ denotes the angle between the TI direction and the downrange direction. The calculation method of $\varepsilon$ is

$$
\varepsilon=a \tan \left(\frac{\Delta H}{\Delta L}\right) .
$$

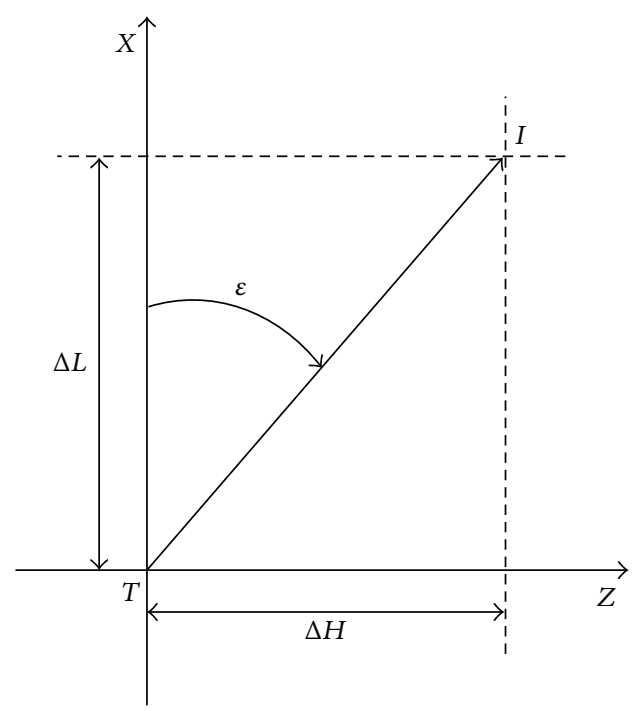

FIgURE 12: Schematic of predictive guidance.

The predictive impact point deviation can be calculated through (10), which is based on the perturbation theory:

$$
\begin{aligned}
\Delta L & =\frac{\partial L}{\partial x} \Delta x+\frac{\partial L}{\partial y} \Delta y+\frac{\partial L}{\partial V_{x}} \Delta V_{x}+\frac{\partial L}{\partial V_{y}} \Delta V_{y} \\
\Delta H & =\frac{\partial H}{\partial z} \Delta z+\frac{\partial H}{\partial V_{z}} \Delta V_{z} \\
\Delta x & =x-x_{0} \\
\Delta y & =y-y_{0} \\
\Delta z & =z-z_{0} \\
\Delta V_{x} & =V_{x}-V_{x 0} \\
\Delta V_{y} & =V_{y}-V_{y 0} \\
\Delta V_{z} & =V_{z}-V_{z 0} .
\end{aligned}
$$

In (10), $L$ is the range function of nominal trajectory, $H$ is the cross range function of nominal trajectory, $x_{0}, y_{0}$, and $z_{0}$ are position vector components of nominal trajectory, $V_{x 0}, V_{y 0}$, and $V_{z 0}$ are velocity vector components of nominal trajectory, $x, y$, and $z$ are position vector components of actual trajectory, and $V_{x}, V_{y}$, and $V_{z}$ are velocity vector components of actual trajectory.

The position and velocity vector components of nominal trajectory $\left(x_{0}, y_{0}, z_{0}, V_{x 0}, V_{y 0}\right.$, and $\left.V_{z 0}\right)$ and differential coefficients $\left(\partial L / \partial x, \partial L / \partial y, \partial L / \partial V_{x}\right.$, and $\left.\partial L / \partial V_{y}\right)$ can be computed and loaded on the onboard computer before flight; the position and velocity vector components of actual trajectory can be acquired by the inertial measurement unit (IMU), or global position system (GPS) receiver. The predictive impact point deviation can be computed online with (10) in flight. 
The canards will deflect according to the predictive impact point deviation; the method of traditional trajectory correction scheme to compute canards control parameters is

$$
\begin{aligned}
\delta_{0} & =k \sqrt{\Delta L^{2}+\Delta H^{2}}, \\
\phi & =\varepsilon .
\end{aligned}
$$

In (11), $k$ is the coefficient, which is set as 0.5 in this paper.

As discussed in Section 3, different canards control phases should be set in ballistic ascending segment and ballistic descending segment to improve the range correction capability. An optimized method of trajectory correction scheme is put forward based on the optimized method of range correction capability:

$$
\begin{aligned}
& \delta_{0}=k \sqrt{\Delta L^{2}+\Delta H^{2}}, \\
& \phi=\pi-\varepsilon \quad t<t_{\text {apex }}, \\
& \phi=\varepsilon \quad t \geq t_{\text {apex }} .
\end{aligned}
$$

As shown in (12), unlike the traditional trajectory correction scheme, the canards control phase of the optimized trajectory correction scheme has different calculation methods in ballistic ascending segment and ballistic descending segment, canards control phase equal $\pi-\varepsilon$ in ballistic ascending segment, and canards control phase equal $\varepsilon$ in ballistic descending segment.

4.2. Optimization of Trajectory Correction Scheme for the Trajectory Tracking Guidance Law. The trajectory tracking guidance law compares the position of actual trajectory to the position of nominal trajectory to get a position error vector in the inertial frame. The trajectory error is converted to the quasibody reference frame using

$$
\begin{aligned}
& {\left[\begin{array}{l}
e_{X} \\
e_{Y} \\
e_{Z}
\end{array}\right]} \\
& =\left[\begin{array}{ccc}
\cos \vartheta \cos \psi & -\sin \vartheta \cos \psi & \sin \psi \\
\sin \vartheta & \cos \phi & 0 \\
-\cos \vartheta \sin \psi & \sin \vartheta \sin \psi & \cos \psi
\end{array}\right]\left[\begin{array}{l}
x-x_{0} \\
y-y_{0} \\
z-z_{0}
\end{array}\right] .
\end{aligned}
$$

In (13), $e_{X}, e_{Y}$, and $e_{Z}$ are trajectory error vector components in the quasibody reference frame.

The magnitude and phase angle of the trajectory error are denoted by $\Gamma$ and $\varepsilon^{\prime}$ and are defined by

$$
\begin{aligned}
\Gamma & =\sqrt{e_{Y}^{2}+e_{Z}^{2}}, \\
\varepsilon^{\prime} & =a \tan \left(\frac{e_{Z}}{e_{Y}}\right) .
\end{aligned}
$$

The canards will deflect according to the trajectory error, and the method of traditional trajectory correction scheme to compute canards control parameters is

$$
\begin{gathered}
\delta_{0}=k^{\prime} \Gamma, \\
\phi=\varepsilon^{\prime} .
\end{gathered}
$$

In (15), $k^{\prime}$ is the coefficient, which is set as 0.5 in this paper.

As discussed in Section 3, different canards control phases should be set in ballistic ascending segment and ballistic descending segment to improve the range correction capability. An optimized method of trajectory correction scheme is put forward based on the optimized method of range correction capability:

$$
\begin{aligned}
& \delta_{0}=k^{\prime} \Gamma \\
& \phi=\pi-\varepsilon^{\prime} \quad t<t_{\mathrm{apex}}, \\
& \phi=\varepsilon^{\prime} \quad t \geq t_{\mathrm{apex}} .
\end{aligned}
$$

\section{Results and Discussion}

To investigate the correction performance of canards and verify the effectiveness of the optimized trajectory correction scheme, some simulations of the guided mortar projectiles have been done by numerical integration of the equations described above using a fourth-order Runge-Kutta algorithm. In these simulations, the trajectory correction performance of the optimized trajectory correction scheme will be compared with the traditional trajectory correction scheme for the predictive guidance law and the trajectory tracking guidance law.

5.1. Results and Discussion for Guided Mortar Projectiles with the Predictive Guidance Law. It can be known from (11) and (12) that the method of traditional trajectory correction scheme to compute canards control parameters is identical with the optimized method if the guidance starts at the trajectory apex time. So the optimized method is used in both cases but with different guidance start times.

Traditional Trajectory Correction Scheme. Equation (12) is adopted to relate the predictive impact point deviation with canards control phase and amplitude, and the control start time is set as the trajectory apex time [11-15].

Optimized Trajectory Correction Scheme. Equation (12) is adopted to relate the predictive impact point deviation with canards control phase and amplitude, and the control start time is set as $5 \mathrm{~s}$. The control start time is set as $5 \mathrm{~s}$ because the control system needs a few time to complete initialization and the GPS receiver needs time to search satellites and start location.

5.1.1. Example Trajectory. An example trajectory is simulated to demonstrate trajectory correction performance. A ballistic case using unperturbed initial conditions shown in Table 1 is used as the nominal trajectory; perturbed initial conditions 
TABLE 1: Initial conditions for example simulation.

\begin{tabular}{lccc}
\hline State & Unit & $\begin{array}{c}\text { Unperturbed } \\
\text { value }\end{array}$ & $\begin{array}{c}\text { Perturbed } \\
\text { value }\end{array}$ \\
\hline launching elevation angle & $\mathrm{deg}$ & 78.86 & 78.26 \\
Launching azimuth angle & $\mathrm{deg}$ & -0.13 & 0.80 \\
Initial velocity & $\mathrm{m} / \mathrm{s}$ & 280 & 285 \\
Wind & $\mathrm{m} / \mathrm{s}$ & 0 & 3 \\
Wind direction & $\mathrm{deg}$ & 0 & 135 \\
\hline
\end{tabular}

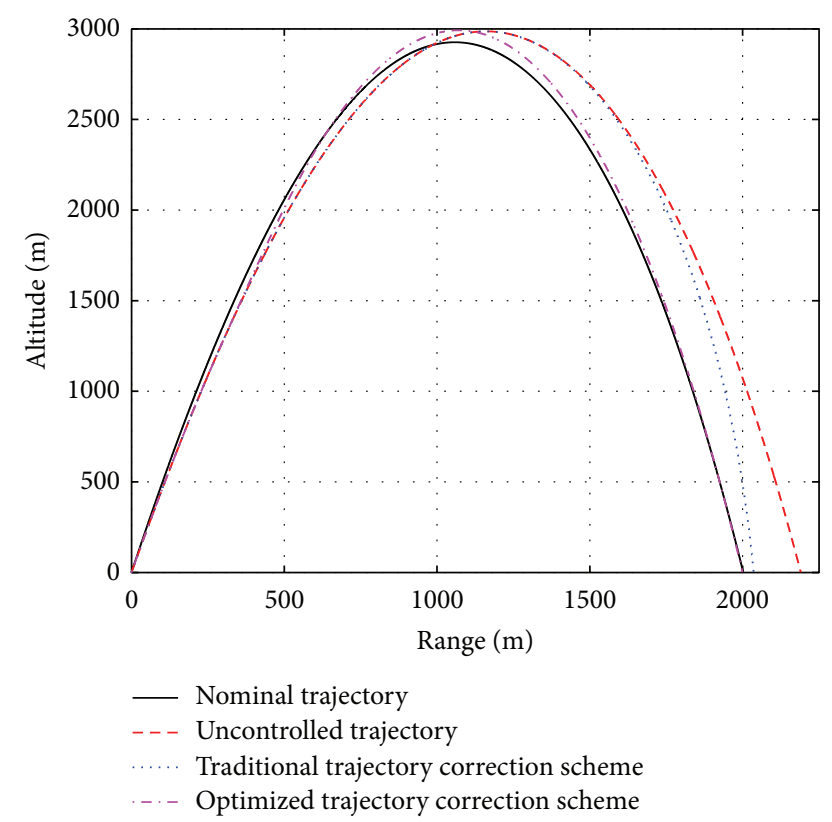

FIGURE 13: Altitude versus range.

shown in Table 1 are used to simulate the uncontrolled trajectory and demonstrate trajectory correction performance.

Figures 13 and 14 compare the trajectory response obtained using the different trajectory correction schemes. The two figures show the nominal trajectory, the uncontrolled trajectory, the controlled trajectory with traditional trajectory correction scheme, and the controlled trajectory with optimized trajectory correction scheme. The longitudinal and horizontal impact point deviations are $190.6 \mathrm{~m}$ and $-171.9 \mathrm{~m}$ in the uncontrolled case, the longitudinal and horizontal impact point deviations of the controlled trajectory with traditional trajectory correction scheme are $52.4 \mathrm{~m}$ and $-44.0 \mathrm{~m}$, the longitudinal and horizontal impact point deviations of the controlled trajectory with optimized trajectory correction scheme are $-0.3 \mathrm{~m}$ and $0.0 \mathrm{~m}$, and the optimized trajectory correction scheme has a better trajectory correction performance.

Figures 15 and 16 show the change of the predicted impact point deviation. It can be known from Figure 15 that the predicted longitudinal impact point deviation before trajectory correction is about $185 \mathrm{~m}$, for the controlled trajectory with traditional trajectory correction scheme, the predicted longitudinal impact point deviation reduces from about

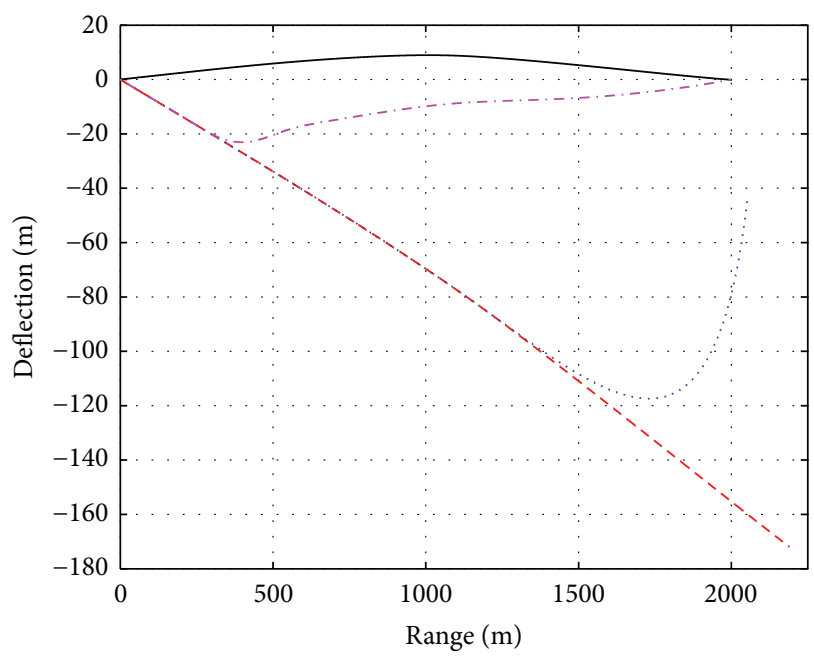

- Nominal trajectory

- - - Uncontrolled trajectory

..... Traditional trajectory correction scheme

-.. Optimized trajectory correction scheme

FIGURE 14: Deflection versus range.

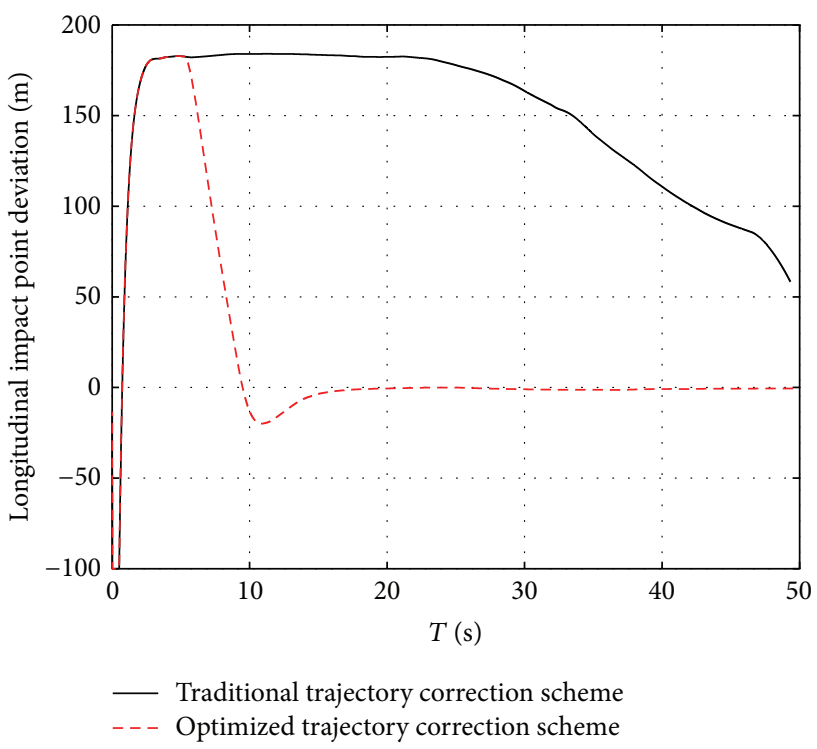

FIGURE 15: The change of longitudinal impact point deviation.

$185 \mathrm{~m}$ at the trajectory apex time to $58.3 \mathrm{~m}$ before impact, and for the controlled trajectory with optimized trajectory correction scheme, the predicted longitudinal impact point deviation starts to reduce at $5 \mathrm{~s}$; it reduces from about $185 \mathrm{~m}$ at $5 \mathrm{~s}$ to about $0 \mathrm{~m}$ at $15 \mathrm{~s}$. It can be known from Figure 16 that the predicted horizontal impact point deviation before trajectory correction is about $-170 \mathrm{~m}$, for the controlled trajectory with traditional trajectory correction scheme, the predicted horizontal impact point deviation reduces from about $-170 \mathrm{~m}$ at the trajectory apex time to $-50 \mathrm{~m}$ before impact, and for the controlled trajectory with optimized trajectory correction scheme, the predicted horizontal impact 


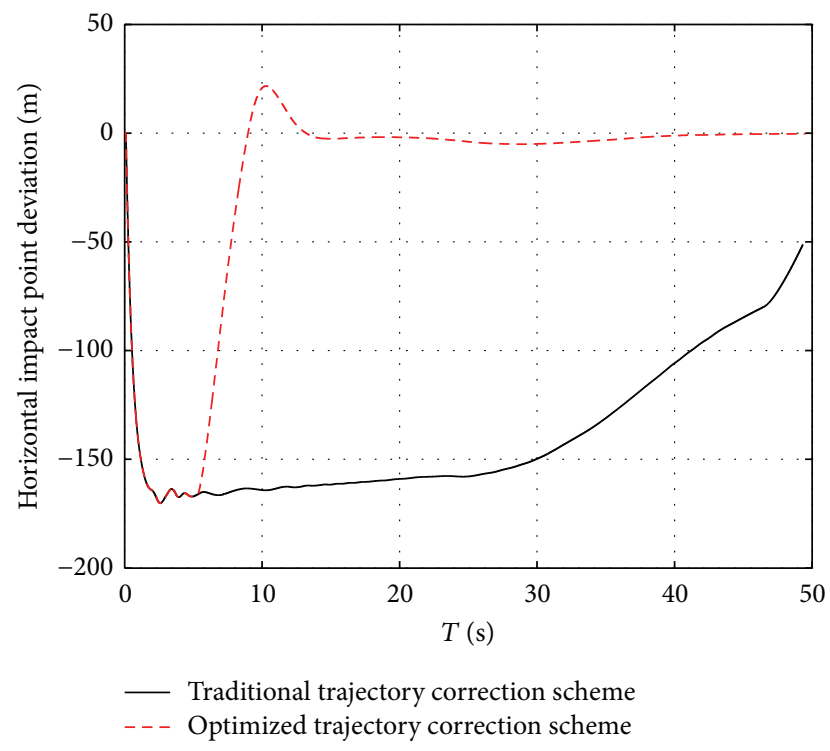

FIgURE 16: The change of horizontal impact point deviation.
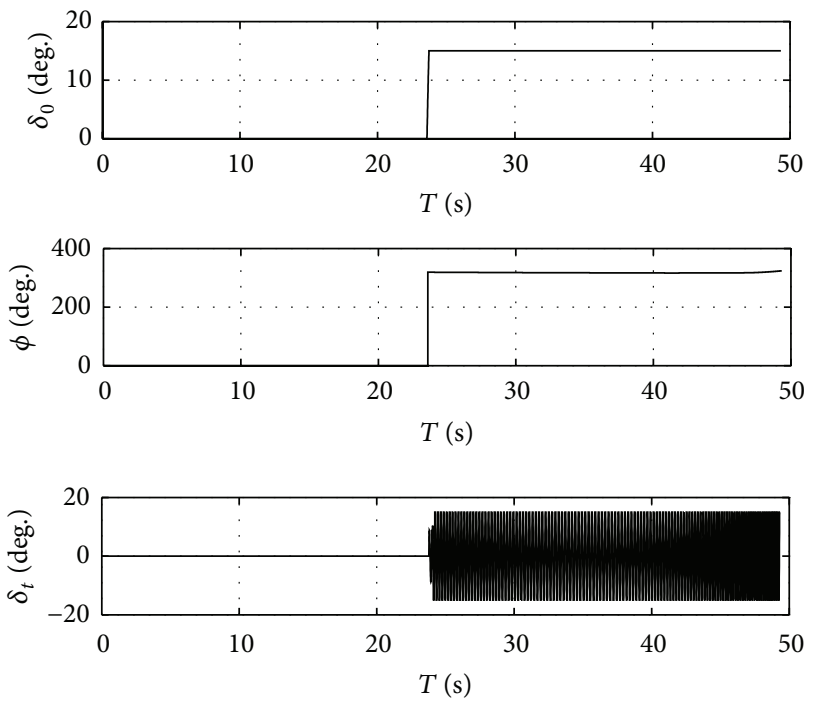

FIGURE 17: The deflection angle and control phase histories of the controlled trajectory with traditional trajectory correction scheme.

point deviation reduces from about $-170 \mathrm{~m}$ at $5 \mathrm{~s}$ to about $0 \mathrm{~m}$ at $13 \mathrm{~s}$. As shown in Figures 15 and 16, the optimized trajectory correction scheme has a bigger trajectory correction capability through taking different calculation methods of canards control phase in ballistic ascending segment and ballistic descending segment, so it can correct the big dispersion error successfully; however, the big dispersion error is not corrected absolutely with the traditional trajectory correction scheme because trajectory correction needs to start after trajectory apex time to avoid range correction capability loss, which leads to a smaller trajectory correction capability.

Figure 17 plots the deflection angle and control phase histories of the controlled trajectory with traditional trajectory correction scheme; the amplitude of canards deflection angle
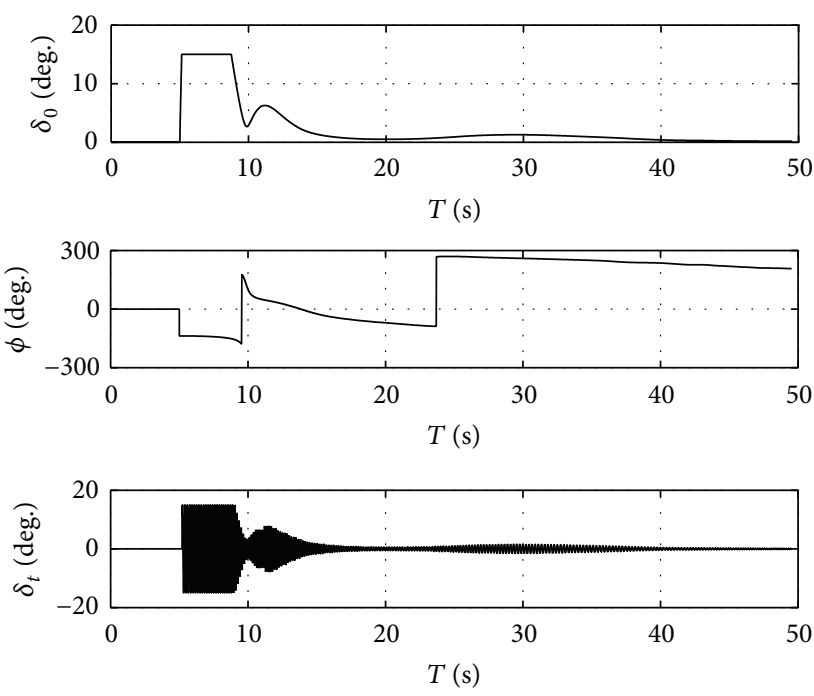

FIGURE 18: The deflection angle and control phase histories of the controlled trajectory with optimized trajectory correction scheme.

TABLE 2: Initial conditions and disturbances.

\begin{tabular}{lccc}
\hline Parameter & Unit & $\begin{array}{c}\text { Mean } \\
\text { value }\end{array}$ & $\begin{array}{c}\text { Std. } \\
\text { deviation }\end{array}$ \\
\hline launching elevation angle & deg & 78.86 & 0.35 \\
Launching azimuth angle & deg & -0.13 & 0.30 \\
Initial velocity & $\mathrm{m} / \mathrm{s}$ & 280 & 3 \\
Wind & $\mathrm{m} / \mathrm{s}$ & 0 & 2 \\
\hline
\end{tabular}

maintains at 15 deg. during the trajectory correction progress because the impact point deviation is not corrected absolutely throughout the trajectory correction progress, and the control phase maintains at about $315 \mathrm{deg}$. Figure 18 plots the deflection angle and control phase histories of the controlled trajectory with optimized trajectory correction scheme; the amplitude of canards deflection angle maintains at about $15 \mathrm{deg}$. during 5 8.7 s and reduces gradually after $8.7 \mathrm{~s}$ due to the decrease of predicted impact point deviation; notably, the control phase maintains at about $-87.5 \mathrm{deg}$. before $23.7 \mathrm{~s}$ and changes to about $267.5 \mathrm{deg}$. at $23.7 \mathrm{~s}$; the quick change of the control phase is determined by the optimized trajectory correction scheme, as shown in (12).

5.1.2. Dispersion Simulations. Dispersion simulations were performed to test control system robustness and effectiveness in eliminating error due to launch perturbations and atmospheric winds. All initial conditions were modeled as Gaussian random variables, with mean values and standard deviations given in Table 2. Wind direction is a uniform random variable between 0 and $2 \pi$. Table 3 lists signal noise and bias standard deviations used in these simulations.

Figure 19 shows the impact point distribution using the Monte Carlo method with a statistical sample of 200 simulations. The cases of the uncontrolled mortar projectiles as well as of the guided mortar projectiles with traditional 


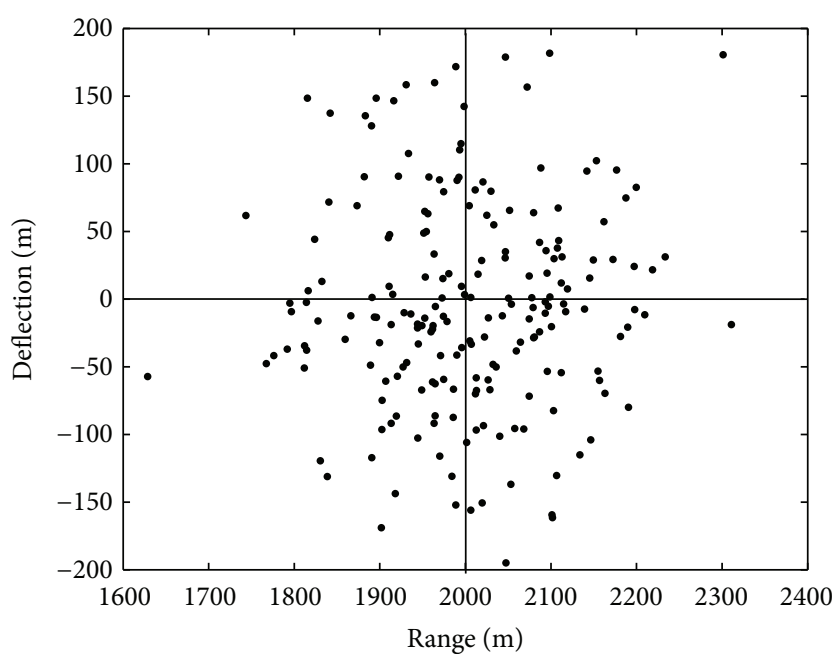

(a) Uncontrolled mortar projectiles

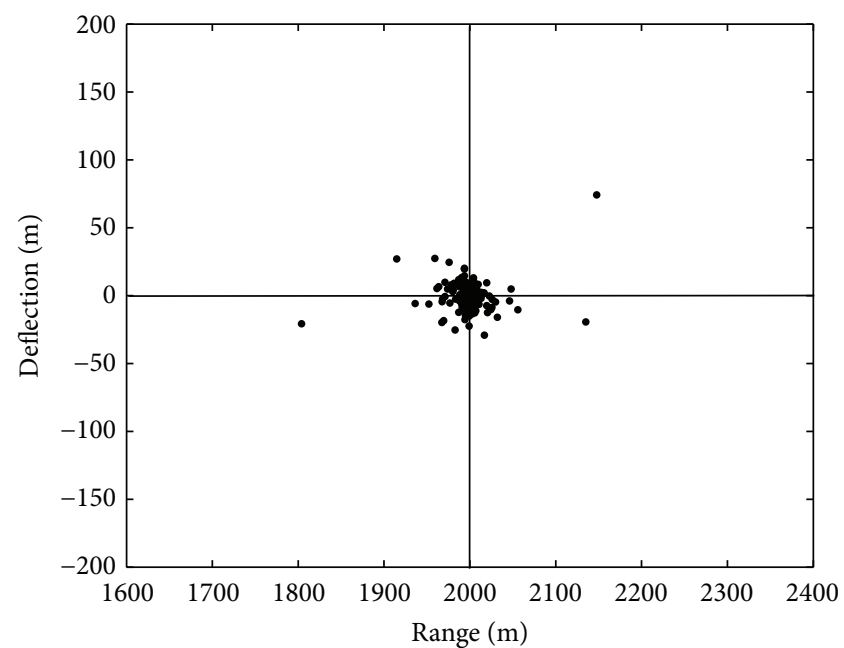

(b) Controlled mortar projectiles with traditional trajectory correction scheme

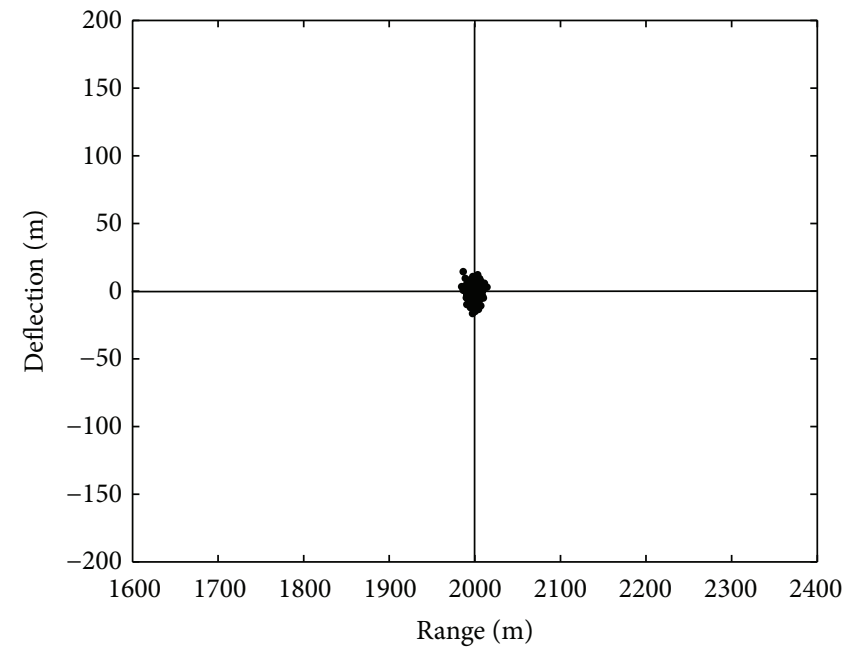

(c) Controlled mortar projectiles with optimized trajectory correction scheme

FIGURE 19: Impact point distribution.

TABLE 3: Signal noise and bias standard deviations.

\begin{tabular}{lccc}
\hline Signal & Unit & Noise std. deviation & Bias std. deviation \\
\hline$x$ & $\mathrm{~m}$ & 8 & 4 \\
$y$ & $\mathrm{~m}$ & 8 & 4 \\
$z$ & $\mathrm{~m}$ & 8 & 4 \\
$v_{x}$ & $\mathrm{~m} / \mathrm{s}$ & 0.4 & 0.2 \\
$v_{y}$ & $\mathrm{~m} / \mathrm{s}$ & 0.4 & 0.2 \\
$v_{z}$ & $\mathrm{~m} / \mathrm{s}$ & 0.4 & 0.2 \\
$\gamma$ & $\mathrm{deg}$ & 5 & 3 \\
\hline
\end{tabular}

trajectory correction scheme and optimized trajectory correction scheme are shown, the impact point distribution of the uncontrolled mortar projectiles is big, as shown in Figure 19(a), and the CEP of the uncontrolled mortar projectiles is $111.0 \mathrm{~m}$. Impact point distribution of the controlled projectiles reduces greatly, as shown in Figures 19(b) and 19(c). The mortar projectiles with the traditional trajectory correction scheme have a CEP of $20.5 \mathrm{~m}$, while the CEP of projectiles with the optimized trajectory correction scheme is $6.2 \mathrm{~m}$. Note that the optimized trajectory correction achieves significantly greater CEP reduction, reflecting the higher trajectory correction authority inherent in this method.

5.2. Results and Discussion for Guided Mortar Projectiles with the Trajectory Tracking Guidance Law. It can be known from (15) and (16) that the method of traditional trajectory correction scheme to compute canards control parameters is identical with the optimized method if the guidance starts at the trajectory apex time. So the optimized method is used in both cases but with different guidance start times.

Traditional Trajectory Correction Scheme. Equation (16) is adopted to relate the predictive impact point deviation with 


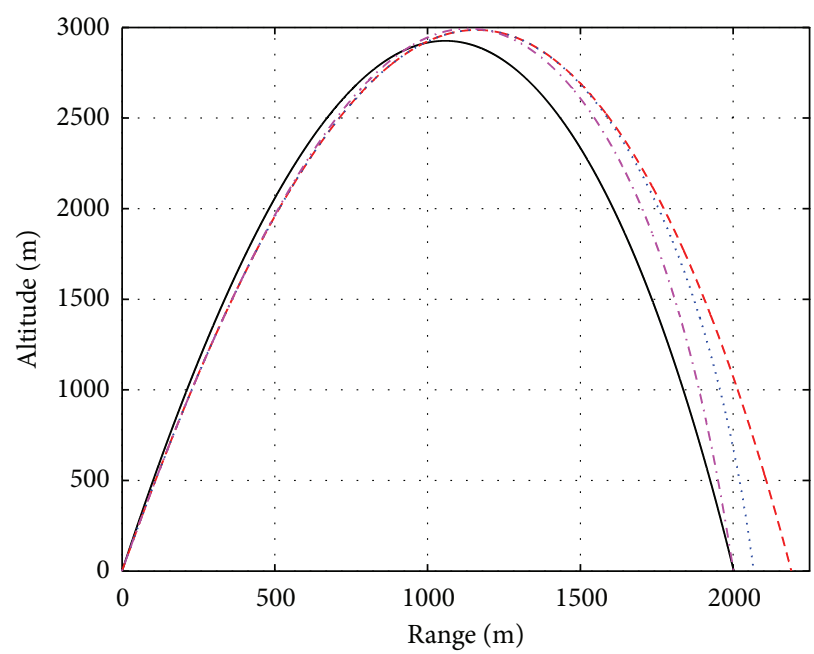

- Nominal trajectory

- - - Uncontrolled trajectory

..... Traditional trajectory correction scheme

-. - Optimized trajectory correction scheme

Figure 20: Altitude versus range.

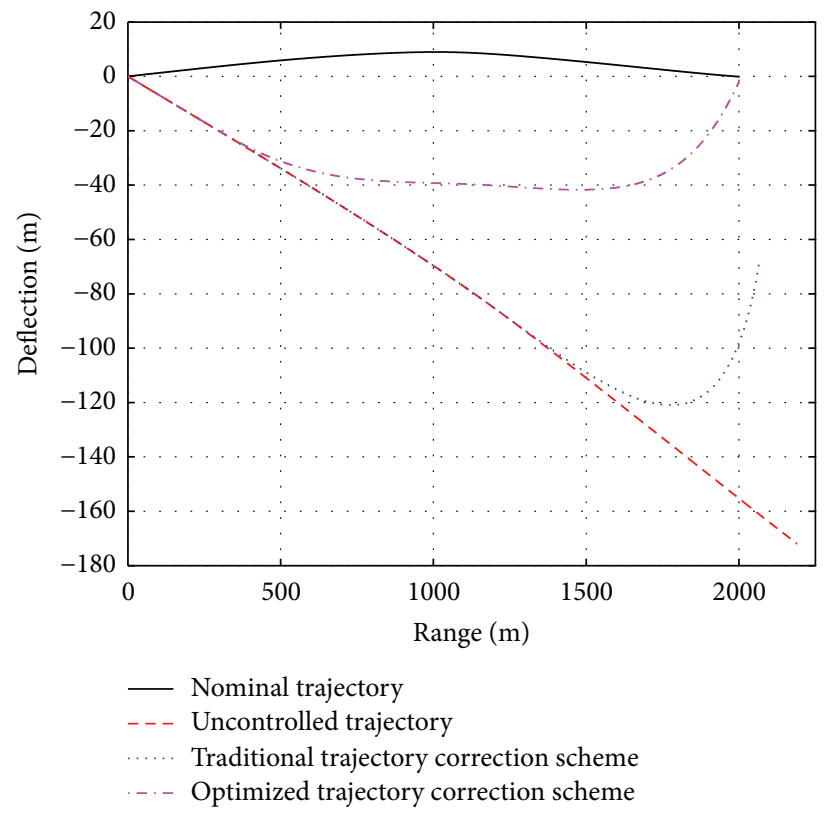

FIGURE 21: Deflection versus range.

canards control phase and amplitude, and the control start time is set as the trajectory apex time [11-15].

Optimized Trajectory Correction Scheme. Equation (16) is adopted to relate the predictive impact point deviation with canards control phase and amplitude, and the control start time is set as $5 \mathrm{~s}$.

5.2.1. Example Trajectory. An example trajectory is simulated to demonstrate trajectory correction performance. A ballistic case using unperturbed initial conditions shown in Table 1 is used as the nominal trajectory; perturbed initial conditions shown in Table 1 are used to simulate the uncontrolled trajectory and demonstrate trajectory correction performance.

Figures 20 and 21 compare the trajectory response obtained using the different trajectory correction schemes. The two figures show the nominal trajectory, the uncontrolled trajectory, the controlled trajectory with traditional trajectory correction scheme, and the controlled trajectory with optimized trajectory correction scheme. The longitudinal and horizontal impact point deviations are $190.6 \mathrm{~m}$ and $-171.9 \mathrm{~m}$ in the uncontrolled case, the longitudinal and horizontal 

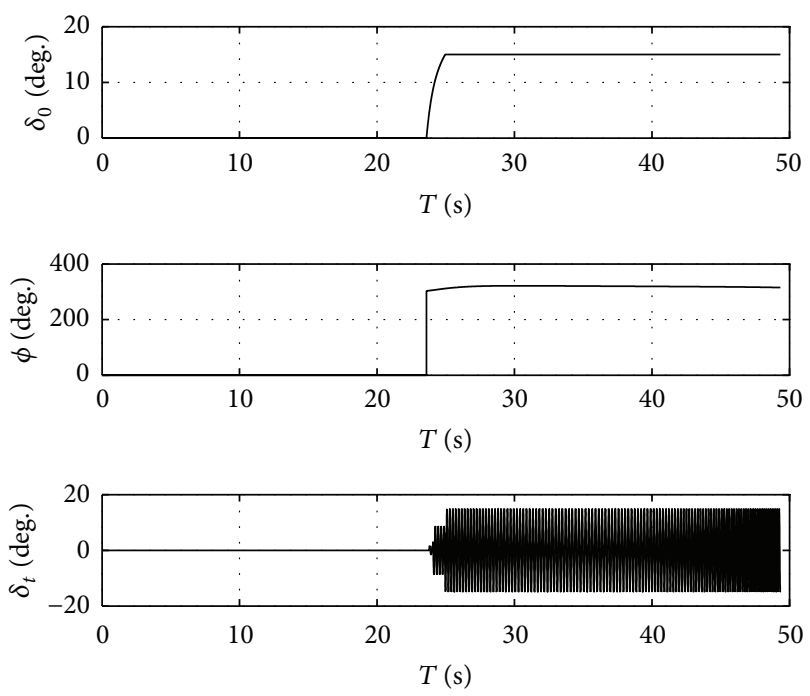

FIGURE 22: The deflection angle and control phase histories of the controlled trajectory with traditional trajectory correction scheme.

impact point deviations of the controlled trajectory with traditional trajectory correction scheme are $67.3 \mathrm{~m}$ and $-67.7 \mathrm{~m}$, the longitudinal and horizontal impact point deviations of the controlled trajectory with optimized trajectory correction scheme are $1.1 \mathrm{~m}$ and $-1.8 \mathrm{~m}$, and the optimized trajectory correction scheme has a better trajectory correction performance.

Figure 22 plots the deflection angle and control phase histories of the controlled trajectory with traditional trajectory correction scheme, the amplitude of canards deflection angle maintains at $15 \mathrm{deg}$. during the trajectory correction progress because the impact point deviation is not corrected absolutely throughout the trajectory correction progress, and the control phase maintains at about $320 \mathrm{deg}$. Figure 23 plots the deflection angle and control phase histories of the controlled trajectory with optimized trajectory correction scheme; the amplitude of canards deflection angle increases from $0 \mathrm{deg}$. to $15 \mathrm{deg}$. during $5 \sim 26 \mathrm{~s}$ and reduces gradually after $37.5 \mathrm{~s}$.

5.2.2. Dispersion Simulations. Dispersion simulations were performed to test control system robustness and effectiveness in eliminating error due to launch perturbations and atmospheric winds. All initial conditions were modeled as Gaussian random variables, with mean values and standard deviations given in Table 2. Wind direction is a uniform random variable between 0 and $2 \pi$. The signal noise and bias standard deviations used in these simulations are listed in Table 3.

Figure 24 shows the impact point distribution using the Monte Carlo method with a statistical sample of 200 simulations. The cases of the uncontrolled mortar projectiles as well as of the guided mortar projectiles with traditional trajectory correction scheme and optimized trajectory correction scheme are shown, the impact point distribution of the uncontrolled mortar projectiles is big, as shown in
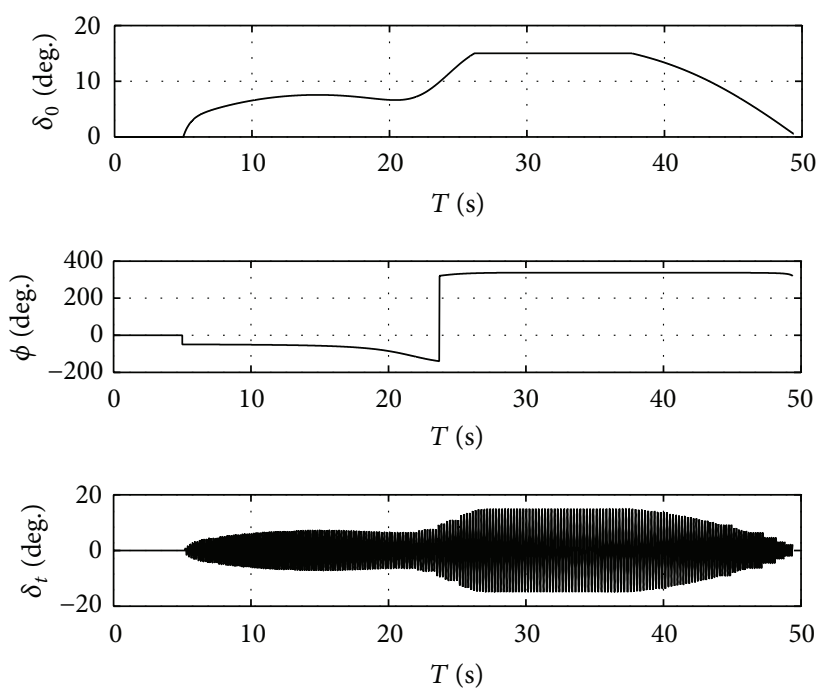

FIGURE 23: The deflection angle and control phase histories of the controlled trajectory with optimized trajectory correction scheme.

Figure 24(a), and the CEP of the uncontrolled mortar projectiles is $109.3 \mathrm{~m}$. Impact point distribution of the controlled projectiles reduces greatly, as shown in Figures 24(b) and 24(c). The mortar projectiles with the traditional trajectory correction scheme have a CEP of $35.1 \mathrm{~m}$, while the CEP of projectiles with the optimized trajectory correction scheme is $16.1 \mathrm{~m}$. Note that the optimized trajectory correction achieves significantly greater CEP reduction, reflecting the higher trajectory correction authority inherent in this method.

\section{Conclusion}

This paper presents an optimized trajectory correction scheme by taking different calculation methods of canards control phase in ballistic ascending segment and ballistic descending segment. Simulation indicates that the optimized trajectory correction scheme can improve the trajectory correction capability greatly. The simulation of an example trajectory and Monte Carlo simulations with the predictive guidance law and the trajectory tracking guidance law were done to investigate the correction performance of canards and verify the effectiveness of the optimized trajectory correction scheme. In the simulations with the predictive guidance law, the result of the example trajectory shows that the optimized trajectory correction scheme has better trajectory correction performance for the ballistics case with big dispersion error, and the result of Monte Carlo simulations shows that the uncontrolled mortar projectiles have a CEP of $111.0 \mathrm{~m}$, the CEP was improved to $20.5 \mathrm{~m}$ with the traditional trajectory correction scheme, and, as a contrast, the CEP was improved to $6.2 \mathrm{~m}$ with the optimized trajectory correction scheme. In the simulations with the trajectory tracking guidance law, the result of the example trajectory shows that the optimized trajectory correction scheme has better trajectory correction performance for the ballistics case with big dispersion error, and the result of 


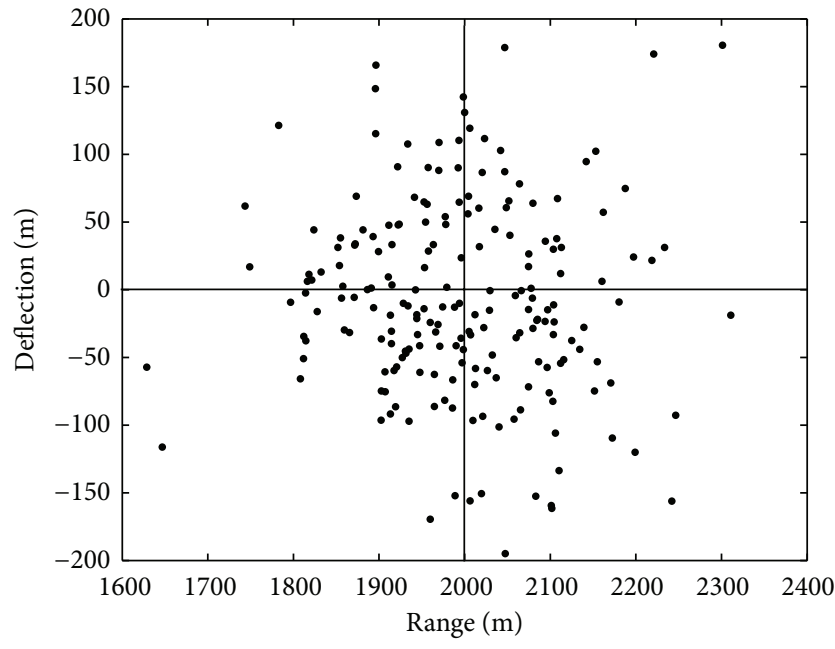

(a) Uncontrolled mortar projectiles

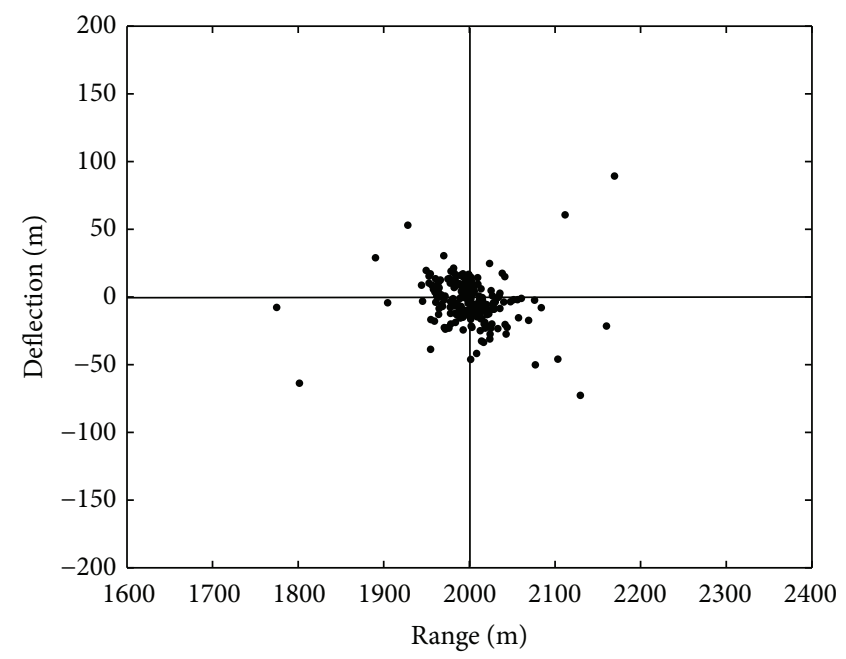

(b) Controlled mortar projectiles with traditional trajectory correction scheme

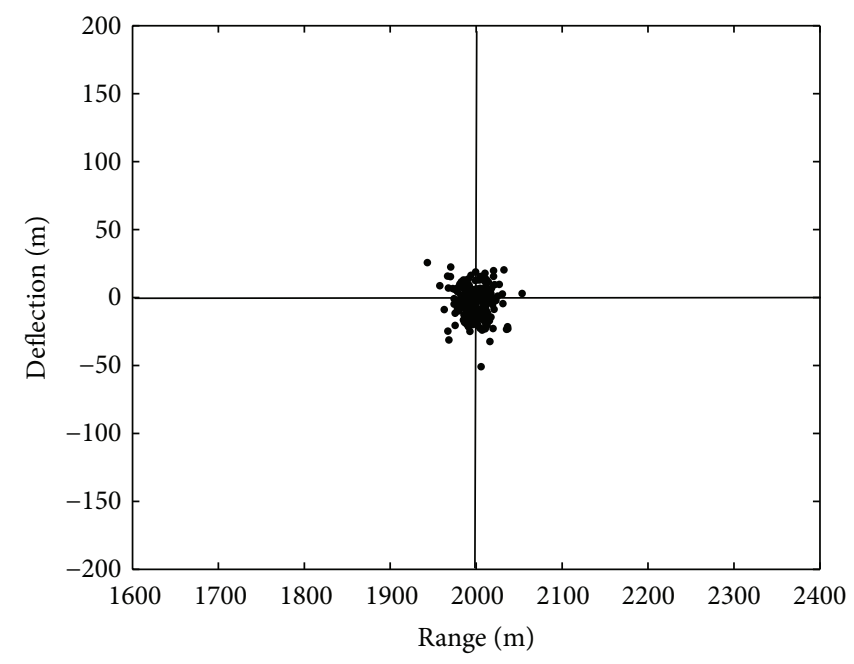

(c) Controlled mortar projectiles with optimized trajectory correction scheme

FIGURE 24: Impact point distribution.

Monte Carlo simulations shows that the uncontrolled mortar projectiles have a CEP of $109.3 \mathrm{~m}$, the CEP was improved to $35.1 \mathrm{~m}$ with the traditional trajectory correction scheme, as a contrast, and the CEP was improved to $16.1 \mathrm{~m}$ with the optimized trajectory correction scheme. The result of the example trajectory and Monte Carlo simulations with the predictive guidance law and the trajectory tracking guidance law testifies the effectiveness of the optimized trajectory correction scheme. The trajectory correction performance of the optimized trajectory correction scheme with other launching elevation angles will be studied in the future work.

\section{Conflict of Interests}

The authors declare that there is no conflict of interests regarding the publication of this paper.

\section{References}

[1] B. Pavkovic, M. Pavic, and D. Cuk, "Enhancing the precision of artillery rockets using pulsejet control systems with active damping," Scientific Technical Review, vol. 62, no. 2, pp. 10-19, 2012.

[2] S. K. Gupta, S. Saxena, A. Singhal, and A. K. Ghosh, “Trajectory correction flight control system using pulsejet on an artillery rocket," Defence Science Journal, vol. 58, no. 1, pp. 15-33, 2008.

[3] M. Gao, Y. Zhang, and S. Yang, "Firing control optimization of impulse thrusters for trajectory correction projectiles," International Journal of Aerospace Engineering, vol. 2015, Article ID 781472, 11 pages, 2015.

[4] J. Rogers and I. Celmins, "Control authority of a mortar using internal translating mass control," U.S. Army Research Lab TRADA503142, 2009.

[5] J. Rogers and M. Costello, "Control authority of a projectile equipped with a controllable internal translating mass," Journal 
of Guidance, Control, and Dynamics, vol. 31, no. 5, pp. 1323-1333, 2008.

[6] M. S. L. Hollis and F. J. Brandon, "Design and analysis of a fuze-configurable range correction device for an artillery projectile," Report ARL-TR-2074, U.S. Army Research Laboratory, Aberdeen Proving Ground, Aberdeen, Md, USA, 1999.

[7] M. S. L. Hollis and F. J. Brandon, "Range correction module for a spin stabilized projectile," U.S. Patent Documents US-5816531, 1998.

[8] E. Gagnon and M. Lauzon, "Course correction fuze concept analysis for in-service $155 \mathrm{~mm}$ spin-stabilized gunnery projectiles," in Proceedings of the AIAA Guidance, Navigation and Control Conference and Exhibit, Honolulu, Hawaii, USA, August 2008.

[9] A. Elsaadany and Y. Wen-Jun, "Accuracy improvement capability of advanced projectile based on course correction fuze concept," The Scientific World Journal, vol. 2014, Article ID 273450, 10 pages, 2014.

[10] S. Theodoulis, F. Sève, and P. Wernert, "Robust gain-scheduled autopilot design for spin-stabilized projectiles with a coursecorrection fuze," Aerospace Science and Technology, vol. 42, pp. 477-489, 2015.

[11] J. Rogers and M. Costello, "Design of a roll-stabilized mortar projectile with reciprocating canards," Journal of Guidance, Control, and Dynamics, vol. 33, no. 4, pp. 1026-1034, 2010.

[12] S. Chang, Z. Wang, and T. Liu, "Analysis of spin-rate property for dual-spin-stabilized projectiles with canards," Journal of Spacecraft and Rockets, vol. 51, no. 3, pp. 958-966, 2014.

[13] G. Cooper, F. Fresconi, and M. Costello, "Flight stability of an asymmetric projectile with activating canards," Journal of Spacecraft and Rockets, vol. 49, no. 1, pp. 130-135, 2012.

[14] J. Spagni, S. Theodoulis, and P. Wernert, "Flight control for a class of $155 \mathrm{~mm}$ spin-stabilized projectile with reciprocating canards," in Proceedings of the AIAA Guidance, Navigation, and Control Conference and Exhibit, Monterey, Calif, USA, August 2012.

[15] S. Theodoulis, V. Gassmann, P. Wernert, L. Dritsas, I. Kitsios, and A. Tzes, "Guidance and control design for a class of spin-stabilized fin-controlled projectiles," Journal of Guidance, Control, and Dynamics, vol. 36, no. 2, pp. 517-531, 2013.

[16] Q. Xingfang, L. Ruixiong, and Z. Yanan, Aerothermodynamics of Missile Flight, Beijing Institute of Technology Press, Beijing, China, 2008.

[17] F. Fresconi and P. Plostins, "Control mechanism strategies for spin-stabilized projectiles," in Proceedings of the 47th AIAA Aerospace Sciences Meeting including the New Horizons Forum and Aerospace Exposition, Orlando, Fla, USA, January 2009.

[18] Y. Wang, W.-D. Song, D. Fang, and Q.-W. Guo, "Guidance and control design for a class of spin-stabilized projectiles with a two-dimensional trajectory correction fuze," International Journal of Aerospace Engineering, vol. 2015, Article ID 908304 , 15 pages, 2015.

[19] T. Jitpraphai, B. Burchett, and M. Costello, "A comparison of different guidance schemes for a direct fire rocket with a pulse jet control mechanism," in Proceedings of the AIAA Atmospheric Flight Mechanics Conference and Exhibit, Montreal, Canada, August 2001. 

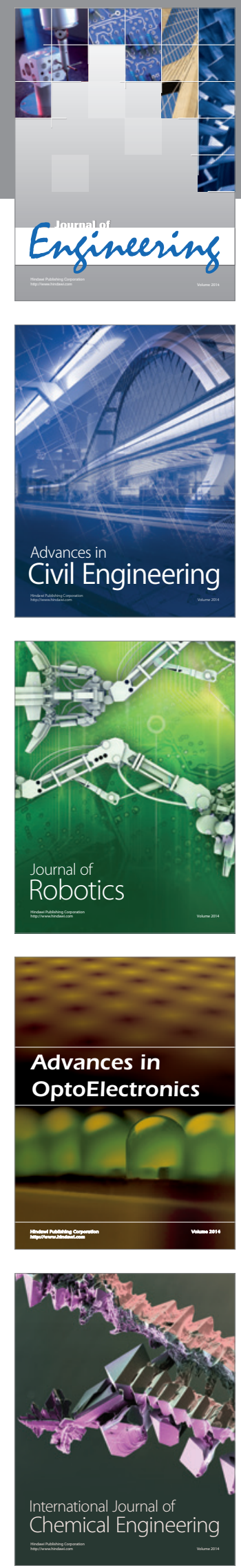



The Scientific World Journal
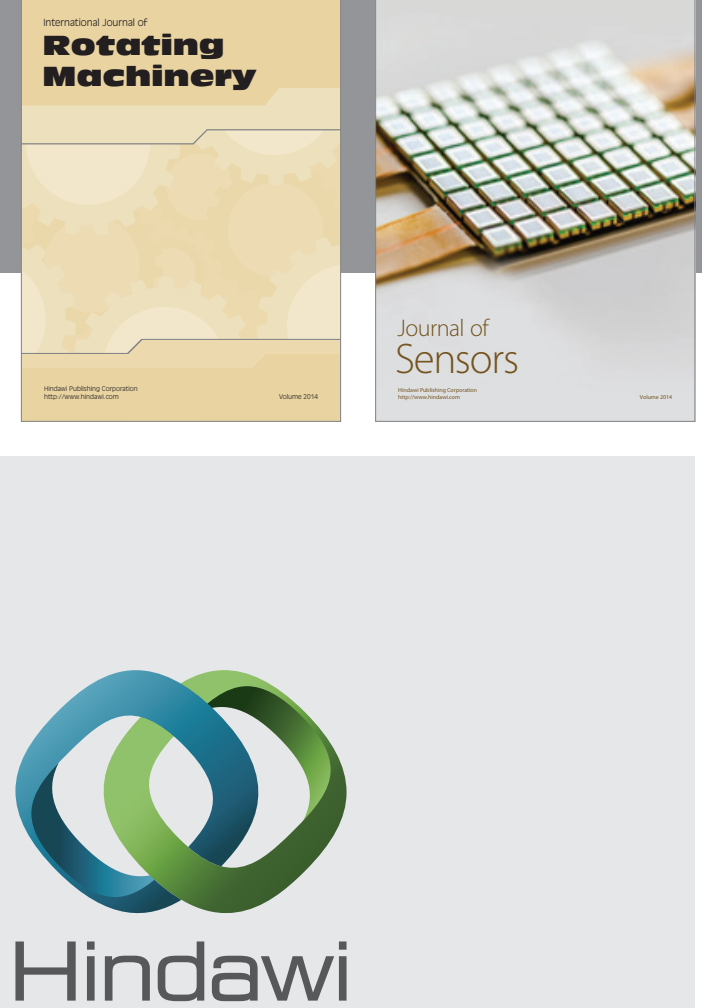

Submit your manuscripts at http://www.hindawi.com
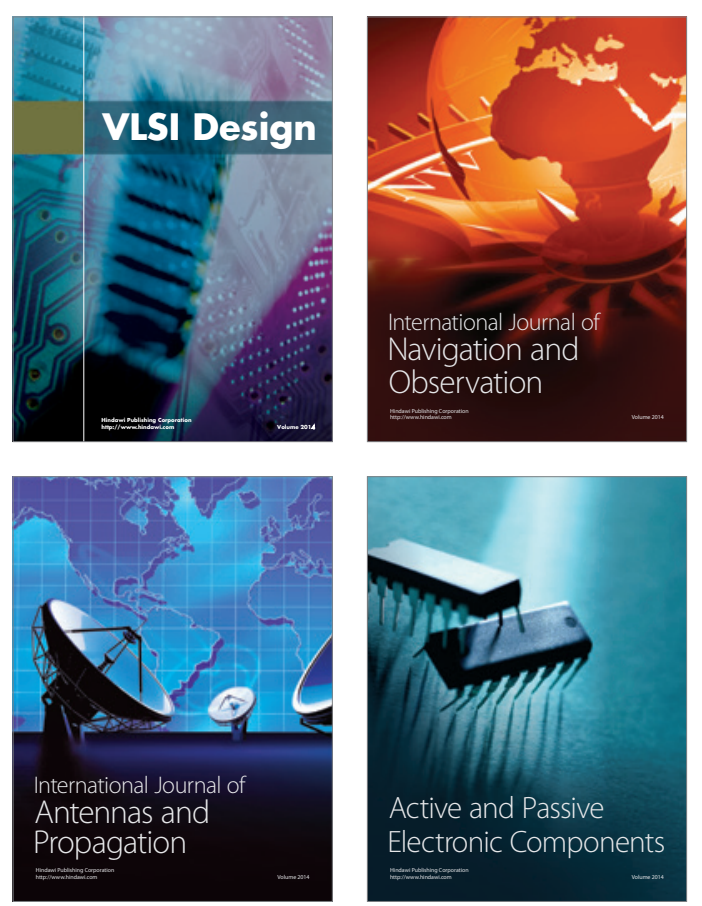
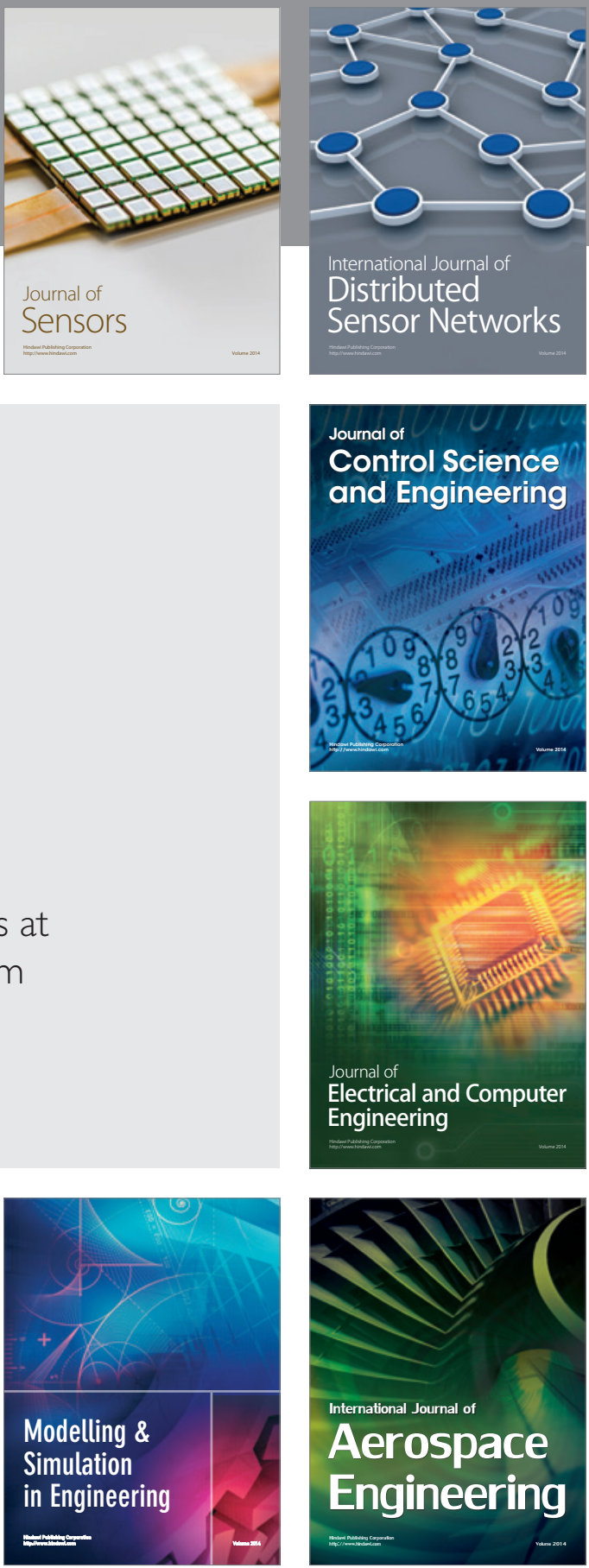

Journal of

Control Science

and Engineering
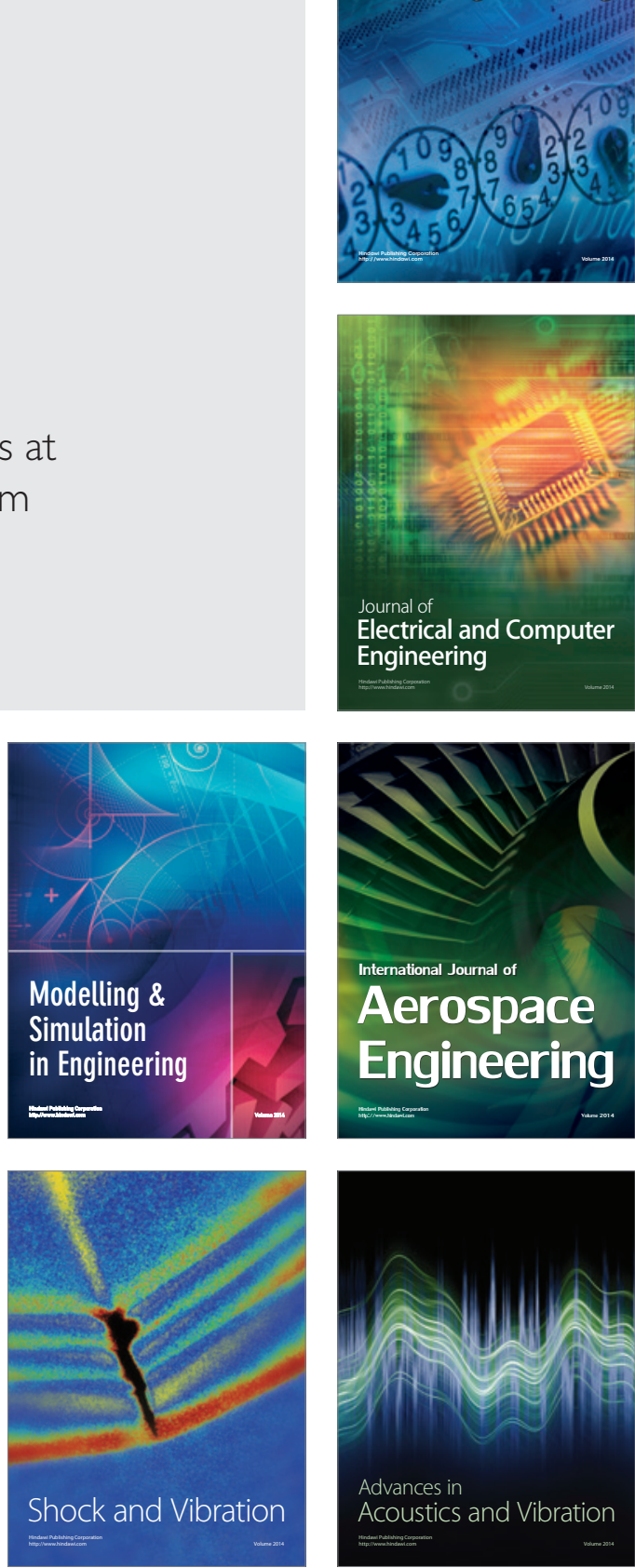